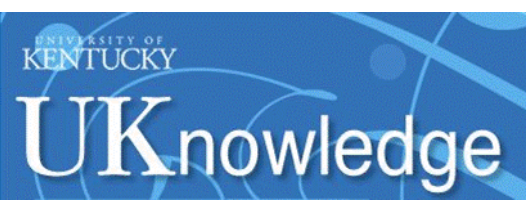

University of Kentucky

UKnowledge

\title{
On the Disappearance of the Broad-Line Region in Low-Luminosity Active Galactic Nuclei
}

\author{
Moshe Elitzur \\ University of Kentucky, moshe@pa.uky.edu \\ Luis C. Ho \\ The Observatoires of the Carnegie Institution of Washington
}

Follow this and additional works at: https://uknowledge.uky.edu/physastron_facpub

Part of the Astrophysics and Astronomy Commons, and the Physics Commons

Right click to open a feedback form in a new tab to let us know how this document benefits you.

\section{Repository Citation}

Elitzur, Moshe and Ho, Luis C., "On the Disappearance of the Broad-Line Region in Low-Luminosity Active Galactic Nuclei" (2010). Physics and Astronomy Faculty Publications. 195.

https://uknowledge.uky.edu/physastron_facpub/195

This Article is brought to you for free and open access by the Physics and Astronomy at UKnowledge. It has been accepted for inclusion in Physics and Astronomy Faculty Publications by an authorized administrator of UKnowledge. For more information, please contact UKnowledge@lsv.uky.edu. 
On the Disappearance of the Broad-Line Region in Low-Luminosity Active Galactic Nuclei

\section{Digital Object Identifier (DOI)}

http://dx.doi.org/10.1088/0004-637X/701/2/L91

Notes/Citation Information

Published in The Astrophysical Journal Letters, v. 701, no. 2, p. L91-L94.

(C) 2010. The American Astronomical Society. All rights reserved.

The copyright holder has granted permission for posting the article here. 


\title{
ON THE DISAPPEARANCE OF THE BROAD-LINE REGION IN LOW-LUMINOSITY ACTIVE GALACTIC NUCLEI
}

\author{
Moshe Elitzur ${ }^{1}$ and Luis C. $\mathrm{Ho}^{2}$ \\ ${ }^{1}$ Department of Physics and Astronomy, University of Kentucky, Lexington, KY 40506-0055, USA; moshe@pa.uky.edu \\ 2 The Observatories of the Carnegie Institution of Washington, 813 Santa Barbara Street, Pasadena, CA 91101, USA; lho@ ociw.edu \\ Received 2009 June 5; accepted 2009 July 21; published 2009 August 4
}

\begin{abstract}
The disk-wind scenario for the broad-line region (BLR) and toroidal obscuration in active galactic nuclei (AGNs) predicts the disappearance of the BLR at low luminosities. In accordance with the model predictions, data from a nearly complete sample of nearby AGNs show that the BLR disappears at luminosities lower than $5 \times 10^{39}\left(M / 10^{7} M_{\odot}\right)^{2 / 3} \mathrm{erg} \mathrm{s}^{-1}$, where $M$ is the black hole mass. The radiative efficiency of accretion onto the black hole is $\lesssim 10^{-3}$ for these sources, indicating that their accretion is advection-dominated.
\end{abstract}

Key words: accretion, accretion disks - galaxies: active - galaxies: Seyfert - quasars: general

\section{INTRODUCTION}

According to the unification model for active galactic nuclei (AGNs), the only difference between type 1 (broad line) and type 2 (narrow line) sources is the observer's orientation with respect to the toroidal dusty obscuration. Directions with clear sight of the central engine and the broad-line region (BLR) yield type 1 sources, while those blocked by the torus from direct view of the BLR result in type 2 objects, where the existence of the hidden BLR is revealed only in polarized light (e.g., Antonucci 1993). However, in spite of the considerable success of the unification scheme there is now clear evidence, recently summarized by Ho (2008), that the BLR is actually missing, and not just hidden, in many low-luminosity AGNs (LLAGNs). These sources have been named "pure" (Tran 2001, 2003) or "true" (Laor 2003) type 2 AGNs. Why does the BLR disappear in LLAGNs?

In another important recent development, the obscuring torus is found to be a smooth continuation of the BLR, not a separate entity. Primary evidence comes from the Suganuma et al. (2006) discovery that the BLR extends outward until the inner boundary of the dusty torus, in agreement with the Netzer \& Laor (1993) proposal that the BLR outer boundary is set by dust sublimation. Additionally, Risaliti et al. (2002) find that X-ray variability caused by the passage of absorbing clouds across the line of sight has a continuous behavior across the timescales generated by the motions of dust-free BLR clouds close to the AGN and the more distant, dusty torus clouds. Together, these observations show that the BLR and the torus are simply the inner and outer regions, respectively, of a single, continuous cloud distribution. Their different radiative signatures merely reflect the change in cloud composition across the dust sublimation radius $R_{\mathrm{d}}$. The inner clouds are dust-free, and their gas is directly exposed to the AGN ionizing continuum; therefore, the gas is atomic and ionized, producing the broad emission lines. The outer clouds are dusty, and so their gas is shielded from the ionizing radiation, and the atomic line emission is quenched. Instead, these clouds are molecular and dusty, obscuring the ultraviolet/ optical emission from the inner regions and emitting infrared radiation. Within this framework, the BLR occupies $r<R_{\mathrm{d}}$ while the torus is simply the region at $r>R_{\mathrm{d}}$, and a more appropriate designation for it is the "toroidal obscuration region" (TOR; Elitzur 2007; Nenkova et al. 2008).
The BLR/TOR structure arises naturally in the disk-wind scenario, first proposed by Emmering et al. (1992). In this model, the two classes of clouds simply correspond to different regions of a clumpy wind coming off the accretion disk rotating around the black hole (see Elitzur \& Shlosman 2006, and references therein). As the clouds rise away from the disk they expand and lose their column density, limiting the vertical scope of broad-line emission and dust obscuration and emission, resulting in a toroidal geometry for both the BLR and the TOR. Although a theory of clumpy disk winds in AGNs is far from full development, an immediate consequence of this scenario is the prediction that the TOR and BLR disappear at low bolometric luminosities (i.e., low accretion rates; Elitzur \& Shlosman 2006; Elitzur 2008). The reason is that, as the mass accretion rate decreases, the mass outflow rate of a disk wind with fixed radial column decreases more slowly and thus cannot be sustained below a certain accretion limit. This unavoidable conclusion follows from simple considerations of mass conservation. However, in the absence of a complete theory for the outflow dynamics, the actual limit and its detailed dependence on the AGN parameters remain undetermined. Presently, the only practical approach is to attempt to extract clues on these unknowns from the analysis of LLAGN data. This is the approach taken here.

\section{THE BLR DISAPPEARANCE}

Denote by $L_{45}=L / 10^{45} \mathrm{erg} \mathrm{s}^{-1}$ the AGN bolometric luminosity and by $M_{7}=M / 10^{7} M_{\odot}$ the black hole mass. From mass conservation, disk outflow can support the column densities required by the BLR and TOR only when the luminosity obeys

$$
L_{45}>\mathrm{CM}_{7}^{2 / 3}
$$

(Elitzur \& Shlosman 2006; Elitzur 2008). The derivation of this result is repeated below in Section 3, where we analyze in detail the assumptions and provide the expression for the numerical coefficient $C$ in terms of system properties other than $L$ and $M$. Replacing the mass with the Eddington luminosity, $L_{\text {Edd }}=1.3 \times 10^{45} M_{7} \mathrm{erg} \mathrm{s}^{-1}$, produces the luminosity lower bound in terms of the Eddington ratio

$$
L_{45}>C_{2}\left(\frac{L_{\text {Edd }}}{L}\right)^{2}
$$



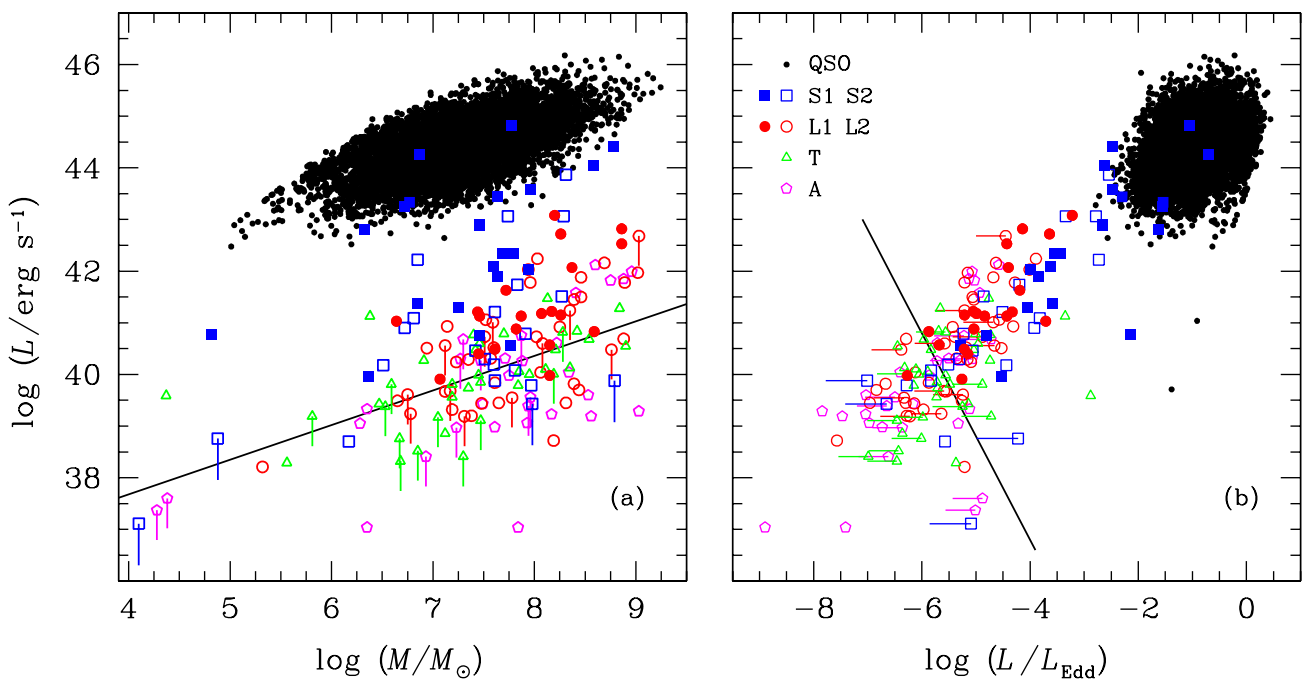

Figure 1. Distribution of (a) black masses, $M$, and (b) Eddington ratios, $L / L_{\text {Edd, }}$,vs. bolometric luminosity, $L$, for objects separated by spectral classification. The bolometric luminosity is based on the 2-10 keV X-ray luminosity, as described in Ho (2009a). The symbols are identified in the legend. Solid and open symbols indicate type 1 and type 2 sources, respectively. "S" = Seyferts, "L" = LINERs, "T" = transition objects, and "A" = absorption-line nuclei. Short line segments denote upper limits. The objects marked as "QSO" refer to the sample of high-luminosity Seyfert 1 nuclei and quasars studied by Greene \& Ho (2007). The solid lines are $\log L=35+\frac{2}{3} \log M$ in panel (a) and $\log L=28.8-2 \log \left(L / L_{\text {Edd }}\right)$ in (b), corresponding to the theoretical predictions given in Equations (1) and (2), respectively.

where $C_{2}=0.59 C^{3}$. In the absence of a detailed theoretical model for the disk wind, our ignorance about the dynamics is contained in the numerical coefficient $C$. The only way to determine whether $C$ may contain additional, indirect dependence on $L$ or $M$ is to compare the bounds in Equations (1) and (2) with observations.

We use data selected from the Palomar spectroscopic survey of 486 nearby galaxies (Ho et al. 1997a, 1997b), the most sensitive and complete sample of LLAGNs available. A critical new development is that the majority of the Palomar galaxies now have reliable estimates of their nuclear luminosities and black hole masses (Ho 2009a, 2009b; Ho et al. 2009). We consider all galaxies classified as Seyferts, low-ionization nuclear emission-line regions (LINERs), and transition objects, which, as argued in Ho (2008, 2009a), are variants of LLAGNs that define a sequence of varying accretion rates. We also include absorption-line nuclei, objects with undetected optical line emission that are good candidates of hosting inactive black holes. High-resolution, hard-X-ray measurements provide the most robust measure of the accretion luminosity in AGNs, especially for weak sources. Of the 277 Palomar galaxies meeting our classification criteria, $166(60 \%)$ have suitable nuclear X-ray (2-10 keV) fluxes or upper limits thereof (Ho 2009a). As discussed in Ho (2009a), these X-ray measurements can be converted to bolometric luminosities with an accuracy of $\sim 0.3$ dex. Importantly, all but one of the galaxies has reliable measurements of the central stellar velocity dispersion (Ho et al. 2009), from which the black hole mass can be estimated to within $\sim 0.3$ dex using the well-established relation between black hole mass and bulge stellar velocity dispersion (Tremaine et al. 2002).

Figure 1 shows the distributions of data points in the $L-M$ and $L-L / L_{\text {Edd }}$ planes. The Palomar LLAGNs are coded according to spectral class and the presence or absence of broad $\mathrm{H} \alpha$ emission (Ho et al. 1997b). For comparison, we also include the sample of low-redshift luminous Seyfert 1 nuclei and quasars studied by Greene \& Ho (2007). It is apparent that, depending on either the black hole mass or Eddington ratio, type 1 sources cease to exist below a certain luminosity. (The only exception is the LINER 1 galaxy NGC 2787, whose luminosity of $10^{39.98} \mathrm{erg} \mathrm{s}^{-1}$ is 0.45 dex below the drawn boundary line.) We interpret this critical luminosity to be the threshold below which the BLR disappears in the disk-wind model. The solid line in each panel represents the bound with the slope taken from either Equation (1) or (2), as appropriate. The line intercepts were adjusted to coincide with the observed type 1-type 2 boundary, yielding $C=4.7 \times 10^{-6}$, i.e., the BLR existence requires $L \gtrsim 5 \times 10^{39} M_{7}^{2 / 3} \mathrm{erg} \mathrm{s}^{-1}$.

\section{THE DISK-WIND CONSTRAINT}

Consider an outflow coming off an annular segment of a disk. Denote by $n(R)$ the density and $v_{z}(R)$ the vertical component of outflow velocity at axial radius $R$ on the disk surface. Then the outflow mass-loss rate is $\dot{M}_{\text {out }}=2 \pi m \int n v_{z} R d R$, where $m$ is the mean atomic mass. Since the dust sublimation radius $R_{\mathrm{d}}$ sets a characteristic scale for both the BLR and TOR, it is convenient to introduce the scaled radius $y=R / R_{\mathrm{d}}$ and the dimensionless velocity profile $u=v_{z}(R) / v_{z}\left(R_{\mathrm{d}}\right)$. Introduce also $N_{R}=\int n(R) d R$, the overall column density along a radius vector on the disk surface. We can describe the radial density variation by the dimensionless profile $\rho=n(R) R_{\mathrm{d}} / N_{R}$, normalized from $\int \rho(y) d y=1$. Then

$$
\dot{M}_{\text {out }}=2 \pi m N_{R} R_{\mathrm{d}} v_{z}\left(R_{\mathrm{d}}\right) I,
$$

where

$$
I=\int \rho(y) u(y) y d y
$$

is a numerical factor of order unity. Written in terms of the radial column density $N_{R}$, this basic expression provides the mass outflow rate of any disk wind, whether smooth or clumpy; in the latter case, $v_{z}$ is the cloud liftoff velocity and the profile $\rho$ describes the variation of number of clouds per unit radial length.

The dust sublimation radius in AGNs is $R_{\mathrm{d}} \simeq 0.4 L_{45}^{1 / 2} \mathrm{pc}$ (Nenkova et al. 2008). The initial outflow velocity, $v_{z}$, is roughly the dispersion velocity of material in the disk-an outflow is established when the ordered motion velocity becomes 
comparable to that of the local random motions. We now make the assumption that this velocity is some fraction $f_{1}$ of the local Keplerian velocity, $v_{z}\left(R_{\mathrm{d}}\right)=f_{1}\left(G M / R_{\mathrm{d}}\right)^{1 / 2}$. Maser observations of the nuclear disk in NGC 3079 show that the velocity dispersion is $\sim 14 \mathrm{~km} \mathrm{~s}^{-1}$ in a small region of strong emission where the Keplerian velocity is $110 \mathrm{~km} \mathrm{~s}^{-1}$ (Kondratko et al. 2005). This observation suggests that $f_{1}$ is probably of order $\sim 0.1$. With this assumption, the mass outflow rate becomes

$$
\dot{M}_{\text {out }}=0.07 f_{1} I N_{22} M_{7}^{1 / 2} L_{45}^{1 / 4} M_{\odot} \mathrm{yr}^{-1},
$$

where $N_{22}=N_{R} / 10^{22} \mathrm{~cm}^{-2}$. This expression applies both for the BLR and TOR portions of the disk wind.

In steady state, $\dot{M}_{\text {out }}$ cannot exceed $\dot{M}_{\text {acc }}$, the mass accretion rate into the BLR/TOR region. A fraction $f_{2}$ of this accreted mass finds its way to the black hole and is converted to the AGN bolometric luminosity with radiative efficiency $\eta$ so that $L=f_{2} \eta \dot{M}_{\mathrm{acc}} c^{2}$. Therefore, $\dot{M}_{\mathrm{acc}}=0.02 L_{45} /\left(\eta f_{2}\right) M_{\odot} \mathrm{yr}^{-1}$ and

$$
\frac{\dot{M}_{\text {out }}}{\dot{M}_{\text {acc }}}=\epsilon \frac{M_{7}^{1 / 2}}{L_{45}^{3 / 4}},
$$

where $\epsilon=3.5 \eta f_{1} f_{2} I N_{22}$. Steady-state mass conservation requires $\dot{M}_{\text {out }} / \dot{M}_{\text {acc }}<1$, yielding Equation (1) with $C=\epsilon^{4 / 3}$. The observational result $C=4.7 \times 10^{-6}$ implies $\eta f_{1} f_{2} I N_{22}=$ $3 \times 10^{-5}$. From photoionization modeling of broad-line emission, the BLR requires a minimal column density of $\sim 10^{22} \mathrm{~cm}^{-2}$ (Netzer 1990), and so $N_{22} \gtrsim 1$. We can reasonably take $I \simeq 1$ and $f_{1} \simeq 0.1$, yielding $f_{2} \eta \simeq 3 \times 10^{-4}$; since the factors $f_{2}$ and $\eta$ do not enter separately, only their product is constrained. A rough estimate of $f_{2}$ can be obtained from self-similar hydromagnetic disk-wind models. The wind applies a back torque on the underlying disk because each magnetic field line can be considered rigid from its footpoint $R$ up to the Alfvén radius $R_{\mathrm{A}}$. Angular momentum conservation implies that $\gamma \equiv\left(R / R_{\mathrm{A}}\right)^{2}$ is roughly the ratio of the mass fluxes for outflow and accretion, or $\gamma \sim \dot{M}_{\text {out }}(R) / \dot{M}_{\text {acc }}(R)$. This ratio is found to be either independent of $R$ (Emmering et al. 1992) or weakly dependent on it (Pelletier \& Pudritz 1992), with a value of $\gamma \simeq 0.1-0.3$. The fraction of $\dot{M}_{\text {acc }}$ that reaches the black hole is $1-\gamma=f_{2}$, therefore $f_{2} \gtrsim 0.7$ and $\eta \simeq 4 \times 10^{-4}$. Such a low radiative efficiency places the BLR disappearance in the domain of radiatively inefficient or advection-dominated accretion flow solutions (for recent reviews, see Narayan 2002; Yuan 2007). A "standard" accretion efficiency $\eta \simeq 0.1$ would require virtually the entire mass accreted into the BLR/TOR to be carried away by the wind, with only a fraction $f_{2} \simeq 3 \times 10^{-3}$ reaching the black hole. This seems unlikely.

\section{DISCUSSION}

Quenching of the BLR/TOR at low accretion rates is an unavoidable consequence of the disk-wind scenario. The reason is that the mass outflow rate is set by the radial column density $N_{R}$ and by the scales of the local radius and Keplerian velocity (Equation (3)). Since the BLR/TOR boundary is set by a fixed value of the radiative flux, the radial scale increases with luminosity as $L^{1 / 2}$ and the velocity scale decreases as $L^{-1 / 4}$. Keeping $N_{R}$ constant, as appropriate for both the BLR and TOR, the mass outflow rate varies with $L$ as $L^{1 / 4}$, more slowly than the linear variation of the accretion rate. Disk-wind mass conservation implies that the minimal column required by an observable BLR cannot be sustained below a certain luminosity. The data verify that this is indeed the case and indicate that the transition to "true" type 2 AGNs occurs in the regime of radiatively inefficient accretion. This adds support to independent indicators that LLAGNs accrete in the advectiondominated mode (Ho 2009a, and references therein).

Bounds similar to Equation (1) were derived by Nicastro (2000) and Laor (2003). Both concluded independently that the BLR should disappear when the luminosity drops below some $L_{\min }=C M^{\alpha}$, where $C$ and $\alpha$ are constants. Replacing $M$ with the Eddington ratio, this condition produces significantly different results depending on the value of $\alpha$ : when $\alpha<1$ it implies a lower limit $L>C_{2}\left(L_{\mathrm{Edd}} / L\right)^{\frac{\alpha}{1-\alpha}}$, where $C_{2}$ is derived from $C$, but when $\alpha>1$ the result is an upper bound $L<C_{2}\left(L / L_{\mathrm{Edd}}\right)^{\frac{\alpha}{\alpha-1}}$; in between, $\alpha=1$ implies that the BLR exists only above some fixed Eddington ratio. ${ }^{3}$ Nicastro suggests a disk outflow origin for the BLR, as here, and uses the disk/ corona model of Witt et al. (1997) to derive $\alpha=7 / 8$, practically indistinguishable from the $\alpha=2 / 3$ found here. However, Nicastro's bound translates into $L>C_{2}\left(L_{\text {Edd }} / L\right)^{7}$, a much steeper boundary than indicated by the data in the $L-L / L_{\mathrm{Edd}}$ plane (Figure 1). Laor suggests the existence of an upper limit to BLR velocities, which leads to $\alpha=2$. Because $\alpha>1$ in this case, it yields $L<C_{2}\left(L / L_{\mathrm{Edd}}\right)^{2}$. This bound is orthogonal to the one plotted in panel (b) of Figure 1 and all AGNs with BLR should lie below it, a prediction that seems in clear conflict with the data. As these examples show, examining the data in the $L-L / L_{\text {Edd }}$ plane amplifies the differences between models; while Equation (2) describes adequately the data in Figure 1(b), the other models do not.

The derivation of the BLR/TOR disappearance presented here is more general than the original one in Elitzur \& Shlosman (2006). Equation (4) holds irrespective of the wind clumpiness and applies equally to the BLR and TOR portions of the disk outflow, with $N_{22}$ the radial column density through each pertinent segment. In both regions, $N_{22} \sim 1$ can be considered a reasonable lower limit for the BLR to generate line emission and the TOR to produce substantial obscuration. The only significant difference between the two cases enters from the factor $I$ (Equation (3)): denoting the inner and outer radii of the disk wind by $R_{\text {in }}$ and $R_{\text {out }}$, respectively, the integration in $I_{\mathrm{BLR}}$ extends from $R_{\text {in }} / R_{\mathrm{d}}$ to 1 , while in $I_{\mathrm{TOR}}$ it is from 1 to $R_{\text {out }} / R_{\mathrm{d}}$. We can reasonably assume Keplerian behavior for the velocity profile, i.e., $u=y^{-1 / 2}$, and parameterize the density profile with a power law, $\rho \propto y^{-q}$, so that the integrand is proportional to $y^{1 / 2-q}$. Depending on $q$, different relations exist between $I_{\mathrm{BLR}}$ and $I_{\mathrm{TOR}}$. The range $1 \leqslant q \leqslant 3 / 2$ yields $I_{\mathrm{TOR}} / I_{\mathrm{BLR}} \approx 1, q<1$ produces $I_{\mathrm{TOR}} / I_{\mathrm{BLR}} \approx\left(R_{\mathrm{Out}} / R_{\mathrm{d}}\right)^{1 / 2}>1$, while $q>3 / 2$ results in $I_{\mathrm{TOR}} / I_{\mathrm{BLR}} \approx\left(R_{\mathrm{in}} / R_{\mathrm{d}}\right)^{1 / 2}<1$. The larger is $I$, the earlier does the quenching of the outflow occur as the luminosity is decreasing. Therefore, depending on the steepness of the radial density profile, the BLR and TOR may disappear either together, when $I_{\mathrm{BLR}} \sim I_{\mathrm{TOR}}$, or in sequence: in AGNs with steep profiles $\left(q>3 / 2 ; I_{\mathrm{BLR}}>I_{\mathrm{TOR}}\right)$ the BLR will disappear first; in shallow ones $\left(q<1 ; I_{\mathrm{TOR}}>I_{\mathrm{BLR}}\right)$ the TOR. Recent clumpy torus modeling of extended IR data sets (Mor et al. 2009; Ramos Almeida et al. 2009) suggests that $q$ can range anywhere from 0 to 3 in different sources, indicating that no single rule applies to all AGNs-sources with and without TOR can exist on either side of the BLR boundary. In any given AGN, the TOR disappearance can be established from the lack of IR emission

\footnotetext{
3 As $\alpha$ increases, the bound is rotating clockwise in the $\log L-\log \left(L / L_{\mathrm{Edd}}\right)$ plane, passing through vertical at $\alpha=1$, with BLR sources located ahead of the rotating boundary.
} 
at a level commensurate with the bolometric luminosity. The absence of a TOR in M87 was demonstrated conclusively by Whysong \& Antonucci (2004), who placed stringent limits on the thermal IR emission, and further solidified by Perlman et al. (2007), who found only a trace of thermal emission that is much weaker than expected from an AGN torus and that can be attributed to neighboring dust. Detailed studies of the IR emission from sources close to the BLR boundary established in Figure 1 can be a powerful tool for probing the density distribution around the black hole in LLAGNs and clarifying the nature of the BLR/TOR disk outflow.

Insightful comments by Roberto Maiolino provided the impetus for this work. M.E. acknowledges partial support by NSF (AST-0807417) and NASA (SSC-40095).

\section{REFERENCES}

Antonucci, R. 1993, ARA\&A, 31, 473

Elitzur, M. 2007, in ASP Conf. Ser. 373, The Central Engine of Active Galactic Nuclei, ed. L. C. Ho \& J.-W. Wang (San Francisco, CA: ASP), 415

Elitzur, M. 2008, New Astron. Rev., 52, 274

Elitzur, M., \& Shlosman, I. 2006, ApJ, 648, L101

Emmering, R. T., Blandford, R. D., \& Shlosman, I. 1992, ApJ, 385, 460

Greene, J. E., \& Ho, L. C. 2007, ApJ, 667, 131

Ho, L. C. 2008, ARA\&A, 46, 475

Ho, L. C. 2009a, ApJ, 699, 626
Ho, L. C. 2009b, ApJ, 699, 638

Ho, L. C., Filippenko, A. V., \& Sargent, W. L. W. 1997a, ApJS, 112, 315

Ho, L. C., Filippenko, A. V., Sargent, W. L. W., \& Peng, C. Y. 1997b, ApJS, 112,391

Ho, L. C., Greene, J. E., Filippenko, A. V., \& Sargent, W. L. W. 2009, ApJS, 183,1

Kondratko, P. T., Greenhill, L. J., \& Moran, J. M. 2005, ApJ, 618, 618

Laor, A. 2003, ApJ, 590, 86

Mor, R., Netzer, H., \& Elitzur, M. 2009, ApJ, submitted (arXiv:0907.1654)

Narayan, R. 2002, in Lighthouses of the Universe: The Most Luminous Celestial Objects and Their Use for Cosmology, ed. M. Gilfanov, R. Sunyaev, \& E. Churazov (Berlin: Springer), 405

Nenkova, M., Sirocky, M. M., Nikutta, R., Ivezić, Ž., \& Elitzur, M. 2008, ApJ, 685,160

Netzer, H. 1990, in Active Galactic Nuclei, ed. T. J. L. Courvoisier \& M. Mayor (Berlin: Springer), 57

Netzer, H., \& Laor, A. 1993, ApJ, 404, L51

Nicastro, F. 2000, ApJ, 530, L65

Pelletier, G., \& Pudritz, R. E. 1992, ApJ, 394, 117

Perlman, E. S., et al. 2007, ApJ, 663, 808

Ramos Almeida, C., et al. 2009, ApJ, in press (arXiv:0906.5368)

Risaliti, G., Elvis, M., \& Nicastro, F. 2002, ApJ, 571, 234

Suganuma, M., et al. 2006, ApJ, 639, 46

Tran, H. D. 2001, ApJ, 554, L19

Tran, H. D. 2003, ApJ, 583, 632

Tremaine, S., et al. 2002, ApJ, 574, 740

Whysong, D., \& Antonucci, R. 2004, ApJ, 602, 116

Witt, H. J., Czerny, B., \& Życki, P. T. 1997, MNRAS, 286, 848

Yuan, F. 2007, in ASP Conf. Ser. 373, The Central Engine of Active Galactic Nuclei, ed. L. C. Ho \& J.-W. Wang (San Francisco, CA: ASP), 95 